\title{
Organometallics Note
}

\section{The First Dimeric Triorganotin Fluoride: Stabilisation by Unsymmetrically Orientated Menthyl Substituents}

\author{
Jens Beckmann, ${ }^{\dagger}$ Dainis Dakternieks, Andrew Duthie* \\ Centre for Chiral and Molecular Technologies, Deakin University, Geelong 3217, Australia
}

\section{Supplementary Material}

Table S1. Atomic coordinates and isotropic displacement parameters (in $\AA^{2}$ ) for $\mathbf{6}$.

Table S2. Anisotropic displacement parameters (in $\AA^{2}$ ) for 6 .

Table S3. Selected geometric parameters $\left(\AA{ }^{\circ}{ }^{\circ}\right)$ for $\mathbf{6}$.

\footnotetext{
$\dagger$ Present address: $\quad$ Institut für Chemie

Freie Universität Berlin

Fabeckstrasse 34-36

Berlin 14195

Germany

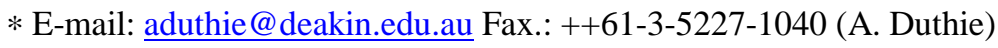


Table S1. Atomic coordinates and isotropic displacement parameters (in $\AA^{2}$ ) for 6 .

\begin{tabular}{|c|c|c|c|c|c|c|}
\hline Atom & Wyck. & Symm. & $\mathbf{x}$ & $\mathbf{y}$ & $\mathbf{z}$ & $\mathbf{U}$ \\
\hline $\mathrm{C} 20$ & $9 b$ & 1 & $0.7840(7)$ & $0.6412(8)$ & $0.39599(18)$ & \\
\hline H9A & $9 b$ & 1 & 0.80380 & 0.58430 & 0.40660 & 0.1790 \\
\hline H9B & $9 b$ & 1 & 0.71540 & 0.63660 & 0.40830 & 0.1790 \\
\hline $\mathrm{H} 9 \mathrm{C}$ & $9 b$ & 1 & 0.85230 & 0.72190 & 0.39820 & 0.1790 \\
\hline C19 & $9 b$ & 1 & $0.7220(6)$ & $0.6989(6)$ & $0.3380(2)$ & \\
\hline H8A & $9 b$ & 1 & 0.70680 & 0.67980 & 0.31280 & 0.1570 \\
\hline H8B & $9 b$ & 1 & 0.78860 & 0.78030 & 0.34070 & 0.1570 \\
\hline $\mathrm{H} 8 \mathrm{C}$ & $9 b$ & 1 & 0.65070 & 0.69250 & 0.34910 & 0.1570 \\
\hline $\mathrm{C} 1$ & $9 b$ & 1 & $0.7447(4)$ & $0.2161(4)$ & $0.17533(12)$ & \\
\hline H11 & $9 b$ & 1 & 0.68540 & 0.13630 & 0.18500 & 0.0720 \\
\hline $\mathrm{C} 2$ & $9 b$ & 1 & $0.7745(5)$ & $0.1953(5)$ & $0.13761(13)$ & \\
\hline $\mathrm{H} 12$ & $9 b$ & 1 & 0.83850 & 0.27420 & 0.12870 & 0.0950 \\
\hline $\mathrm{C} 4$ & $9 b$ & 1 & $0.9415(5)$ & $0.1622(5)$ & $0.16195(17)$ & \\
\hline H14A & $9 b$ & 1 & 0.97330 & 0.10690 & 0.16230 & 0.1110 \\
\hline H14B & $9 b$ & 1 & 1.00350 & 0.23870 & 0.15190 & 0.1110 \\
\hline $\mathrm{C} 3$ & $9 b$ & 1 & $0.8288(6)$ & $0.1095(5)$ & $0.13793(19)$ & \\
\hline H13A & $9 b$ & 1 & 0.76870 & 0.03020 & 0.14690 & 0.1210 \\
\hline H13B & $9 b$ & 1 & 0.85030 & 0.09970 & 0.11350 & 0.1210 \\
\hline $\mathrm{C} 5$ & $9 b$ & 1 & $0.9136(5)$ & $0.1827(5)$ & $0.20038(16)$ & \\
\hline H15 & $9 b$ & 1 & 0.85260 & 0.10400 & 0.21030 & 0.1040 \\
\hline $\mathrm{F} 1$ & $3 a$ & -3 & $2 / 3$ & $1 / 3$ & $0.23605(9)$ & \\
\hline $\mathrm{F} 2$ & $3 a$ & -3 & $2 / 3$ & $1 / 3$ & $0.36643(12)$ & \\
\hline $\mathrm{C} 8$ & $9 b$ & 1 & $0.6672(7)$ & $0.1522(8)$ & $0.11126(19)$ & \\
\hline $\mathrm{H} 18$ & $9 b$ & 1 & 0.63270 & 0.20560 & 0.11390 & 0.1490 \\
\hline C6 & $9 b$ & 1 & $0.8584(5)$ & $0.2663(5)$ & $0.19993(14)$ & \\
\hline H16A & $9 b$ & 1 & 0.91900 & 0.34610 & 0.19140 & 0.0800 \\
\hline H16B & $9 b$ & 1 & 0.83620 & 0.27500 & 0.22440 & 0.0800 \\
\hline $\mathrm{C} 7$ & $9 b$ & 1 & $1.0245(9)$ & $0.2335(11)$ & $0.2246(2)$ & \\
\hline H17A & $9 b$ & 1 & 1.00230 & 0.24280 & 0.24870 & 0.2320 \\
\hline H17B & $9 b$ & 1 & 1.08540 & 0.31120 & 0.21550 & 0.2320 \\
\hline $\mathrm{H} 17 \mathrm{C}$ & $9 b$ & 1 & 1.05600 & 0.17890 & 0.22480 & 0.2320 \\
\hline C9 & $9 b$ & 1 & $0.5703(8)$ & $0.0271(9)$ & $0.1169(3)$ & \\
\hline H19A & $9 b$ & 1 & 0.53480 & 0.01990 & 0.14030 & 0.2620 \\
\hline H19B & $9 b$ & 1 & 0.60330 & -0.02630 & 0.11550 & 0.2620 \\
\hline H19C & $9 b$ & 1 & 0.50920 & 0.00540 & 0.09870 & 0.2620 \\
\hline $\mathrm{C} 10$ & $9 b$ & 1 & $0.7062(11)$ & $0.1578(14)$ & $0.0728(2)$ & \\
\hline $\mathrm{H} 20 \mathrm{~A}$ & $9 b$ & 1 & 0.63730 & 0.13180 & 0.05720 & 0.3220 \\
\hline $\mathrm{H} 20 \mathrm{~B}$ & $9 b$ & 1 & 0.73960 & 0.10530 & 0.06970 & 0.3220 \\
\hline $\mathrm{H} 20 \mathrm{C}$ & $9 b$ & 1 & 0.76660 & 0.23970 & 0.06690 & 0.3220 \\
\hline Sn1 & $3 a$ & -3 & $2 / 3$ & $1 / 3$ & $0.18208(1)$ & \\
\hline $\operatorname{Sn} 2$ & $3 a$ & -3 & $2 / 3$ & $1 / 3$ & $0.31287(1)$ & \\
\hline $\mathrm{C} 16$ & $9 b$ & 1 & $0.9131(4)$ & $0.5116(4)$ & $0.28306(14)$ & \\
\hline H6A & $9 b$ & 1 & 0.93950 & 0.46680 & 0.29820 & 0.0730 \\
\hline H6B & $9 b$ & 1 & 0.88280 & 0.46720 & 0.26060 & 0.0730 \\
\hline $\mathrm{C} 15$ & $9 b$ & 1 & $1.0223(4)$ & $0.6368(4)$ & $0.27486(15)$ & \\
\hline H5 & $9 b$ & 1 & 0.99470 & 0.67970 & 0.25890 & 0.0900 \\
\hline $\mathrm{C} 18$ & $9 b$ & 1 & $0.7532(5)$ & $0.6107(5)$ & $0.35600(14)$ & \\
\hline $\mathrm{H} 7$ & $9 b$ & 1 & 0.67950 & 0.53070 & 0.35510 & 0.0890 \\
\hline $\mathrm{C} 11$ & $9 b$ & 1 & $0.8082(3)$ & $0.5185(3)$ & $0.30246(11)$ & \\
\hline H1 & $9 b$ & 1 & 0.77590 & 0.55520 & 0.28580 & 0.0640 \\
\hline $\mathrm{C} 12$ & $9 b$ & 1 & $0.8532(4)$ & $0.5977(3)$ & $0.33649(11)$ & \\
\hline $\mathrm{H} 2$ & $9 b$ & 1 & 0.87960 & 0.55600 & 0.35340 & 0.0720 \\
\hline $\mathrm{C} 17$ & $9 b$ & 1 & $1.1212(6)$ & $0.6241(7)$ & $0.2549(2)$ & \\
\hline $\mathrm{H} 10 \mathrm{~A}$ & $9 b$ & 1 & 1.08820 & 0.57880 & 0.23300 & 0.1820 \\
\hline H10B & $9 b$ & 1 & 1.14980 & 0.58230 & 0.27000 & 0.1820 \\
\hline $\mathrm{H} 10 \mathrm{C}$ & $9 b$ & 1 & 1.18760 & 0.70330 & 0.24910 & 0.1820 \\
\hline $\mathrm{C} 13$ & $9 b$ & 1 & $0.9662(5)$ & $0.7200(4)$ & $0.32762(16)$ & \\
\hline $\mathrm{H} 3 \mathrm{~A}$ & $9 b$ & 1 & 0.94280 & 0.76540 & 0.31170 & 0.1060 \\
\hline H3B & $9 b$ & 1 & 0.99690 & 0.76620 & 0.34980 & 0.1060 \\
\hline
\end{tabular}




$\begin{array}{lllllll}\text { C14 } & 9 b & 1 & 1.0657(5) & 0.7084(5) & 0.31010(17) & \\ \text { H4A } & 9 b & 1 & 1.09330 & 0.66750 & 0.32650 & 0.1090 \\ \text { H4B } & 9 b & 1 & 1.13380 & 0.78870 & 0.30520 & 0.1090\end{array}$


Table S2. Anisotropic displacement parameters (in $\AA^{2}$ ) for 6 .

$\begin{array}{lllllll}\text { Atom } & \boldsymbol{U}_{\mathbf{1 1}} & \boldsymbol{U}_{\mathbf{2 2}} & \boldsymbol{U}_{\mathbf{3 3}} & \boldsymbol{U}_{\mathbf{1 2}} & \boldsymbol{U}_{\mathbf{1 3}} & \boldsymbol{U}_{\mathbf{2 3}} \\ \text { C20 } & 0.144(6) & 0.166(7) & 0.078(4) & 0.100(6) & -0.020(4) & -0.043(4) \\ \text { C19 } & 0.126(5) & 0.091(4) & 0.127(6) & 0.078(4) & 0.000(4) & -0.006(4) \\ \text { C1 } & 0.066(2) & 0.057(2) & 0.061(2) & 0.034(2) & 0.006(2) & -0.0074(19) \\ \text { C2 } & 0.085(3) & 0.092(3) & 0.067(3) & 0.050(3) & -0.001(2) & -0.025(2) \\ \text { C4 } & 0.092(4) & 0.095(4) & 0.113(5) & 0.065(3) & 0.023(3) & 0.000(3) \\ \text { C3 } & 0.112(4) & 0.095(4) & 0.111(5) & 0.063(4) & 0.016(4) & -0.027(3) \\ \text { C5 } & 0.096(4) & 0.099(4) & 0.093(4) & 0.071(3) & 0.022(3) & 0.020(3) \\ \text { F1 } & 0.0546(11) & 0.0546(11) & 0.0368(19) & 0.0273(5) & 0.00000 & 0.00000 \\ \text { F2 } & 0.0787(17) & 0.0787(17) & 0.056(2) & 0.0394(9) & 0.00000 & 0.00000 \\ \text { C8 } & 0.128(5) & 0.177(7) & 0.098(5) & 0.100(6) & -0.045(4) & -0.080(5) \\ \text { C6 } & 0.070(3) & 0.081(3) & 0.061(3) & 0.047(3) & 0.007(2) & 0.002(2) \\ \text { C7 } & 0.173(8) & 0.269(12) & 0.117(7) & 0.182(9) & -0.032(6) & -0.027(7) \\ \text { C9 } & 0.126(6) & 0.172(9) & 0.240(13) & 0.085(7) & -0.087(8) & -0.144(9) \\ \text { C10 } & 0.245(13) & 0.385(18) & 0.089(6) & 0.214(14) & -0.057(7) & -0.118(9) \\ \text { Sn1 } & 0.05073(19) & 0.05073(19) & 0.0384(2) & 0.02536(10) & 0.00000 & 0.00000 \\ \text { Sn2 } & 0.04634(18) & 0.04634(18) & 0.0565(3) & 0.02317(9) & 0.00000 & 0.00000 \\ \text { C16 } & 0.050(2) & 0.051(2) & 0.066(3) & 0.0151(19) & 0.002(2) & -0.005(2) \\ \text { C15 } & 0.064(3) & 0.060(2) & 0.082(3) & 0.016(2) & 0.000(2) & 0.007(2) \\ \text { C18 } & 0.094(3) & 0.073(3) & 0.071(3) & 0.053(3) & -0.015(3) & -0.015(2) \\ \text { C11 } & 0.056(2) & 0.0443(18) & 0.056(2) & 0.0231(16) & -0.0122(17) & -0.0024(16) \\ \text { C12 } & 0.069(2) & 0.054(2) & 0.058(2) & 0.0324(19) & -0.0169(19) & -0.0082(18) \\ \text { C17 } & 0.061(3) & 0.117(5) & 0.151(7) & 0.018(3) & 0.026(4) & 0.006(5) \\ \text { C13 } & 0.092(4) & 0.059(3) & 0.098(4) & 0.024(3) & -0.019(3) & -0.022(3) \\ \text { C14 } & 0.067(3) & 0.067(3) & 0.100(4) & 0.005(2) & -0.017(3) & -0.013(3) \\ & & & & & & \end{array}$


Table S3. Selected geometric parameters $\left(\AA^{\AA},{ }^{\circ}\right)$ for 6 .

\begin{tabular}{|c|c|c|c|}
\hline $\mathrm{C} 20-\mathrm{C} 18$ & $1.530(8)$ & $\mathrm{C} 8-\mathrm{C} 10$ & $1.497(11)$ \\
\hline $\mathrm{C} 19-\mathrm{C} 18$ & $1.517(11)$ & $\mathrm{Sn} 1-\mathrm{C} 1^{\mathrm{i}}$ & $2.179(1)$ \\
\hline $\mathrm{C} 1-\mathrm{C} 2$ & $1.504(7)$ & $\mathrm{Sn} 1-\mathrm{C} 1^{\mathrm{ii}}$ & $2.179(1)$ \\
\hline $\mathrm{C} 1-\mathrm{C} 6$ & $1.550(7)$ & $\mathrm{Sn} 2-\mathrm{C} 11^{\mathrm{ii}}$ & $2.165(4)$ \\
\hline $\mathrm{C} 1-\mathrm{Sn} 1$ & $2.178(6)$ & $\mathrm{Sn} 2-\mathrm{C} 11$ & $2.166(3)$ \\
\hline $\mathrm{C} 2-\mathrm{C} 8$ & $1.537(10)$ & $\mathrm{Sn} 2-\mathrm{C} 11^{\mathrm{i}}$ & $2.166(4)$ \\
\hline $\mathrm{C} 2-\mathrm{C} 3$ & $1.555(11)$ & $\mathrm{C} 16-\mathrm{C} 15$ & $1.531(5)$ \\
\hline $\mathrm{C} 4-\mathrm{C} 5$ & $1.519(9)$ & $\mathrm{C} 16-\mathrm{C} 11$ & $1.555(7)$ \\
\hline $\mathrm{C} 4-\mathrm{C} 3$ & $1.527(9)$ & $\mathrm{C} 15-\mathrm{C} 14$ & $1.527(8)$ \\
\hline $\mathrm{C} 5-\mathrm{C} 7$ & $1.516(11)$ & $\mathrm{C} 15-\mathrm{C} 17$ & $1.535(11)$ \\
\hline $\mathrm{C} 5-\mathrm{C} 6$ & $1.539(11)$ & $\mathrm{C} 18-\mathrm{C} 12$ & $1.541(9)$ \\
\hline $\mathrm{F} 1-\mathrm{Sn} 1$ & $1.997(3)$ & $\mathrm{C} 11-\mathrm{C} 12$ & $1.533(5)$ \\
\hline $\mathrm{F} 2-\mathrm{Sn} 2$ & $1.981(4)$ & $\mathrm{C} 12-\mathrm{C} 13$ & $1.535(5)$ \\
\hline $\mathrm{C} 8-\mathrm{C} 9$ & $1.460(11)$ & $\mathrm{C} 13-\mathrm{C} 14$ & $1.493(10)$ \\
\hline $\mathrm{C} 2-\mathrm{C} 1-\mathrm{C} 6$ & $110.63(39)$ & $\mathrm{F} 2-\mathrm{Sn} 2-\mathrm{C} 11$ & $100.25(11)$ \\
\hline $\mathrm{C} 2-\mathrm{C} 1-\mathrm{Sn} 1$ & $117.88(36)$ & $\mathrm{C} 11^{\mathrm{ii}}-\mathrm{Sn} 2-\mathrm{C} 11$ & $116.93(13)$ \\
\hline $\mathrm{C} 6-\mathrm{C} 1-\mathrm{Sn} 1$ & $107.51(38)$ & $\mathrm{F} 2-\mathrm{Sn} 2-\mathrm{C} 11^{\mathrm{i}}$ & $100.24(5)$ \\
\hline $\mathrm{C} 1-\mathrm{C} 2-\mathrm{C} 8$ & $113.24(44)$ & $\mathrm{C} 11^{\mathrm{ii}}-\mathrm{Sn} 2-\mathrm{C} 11^{\mathrm{i}}$ & $116.91(5)$ \\
\hline $\mathrm{C} 1-\mathrm{C} 2-\mathrm{C} 3$ & $110.82(48)$ & $\mathrm{C} 11-\mathrm{Sn} 2-\mathrm{C} 11^{\mathrm{i}}$ & $116.88(10)$ \\
\hline $\mathrm{C} 8-\mathrm{C} 2-\mathrm{C} 3$ & $112.37(61)$ & $\mathrm{C} 15-\mathrm{C} 16-\mathrm{C} 11$ & $112.97(41)$ \\
\hline $\mathrm{C} 5-\mathrm{C} 4-\mathrm{C} 3$ & $111.82(52)$ & $\mathrm{C} 14-\mathrm{C} 15-\mathrm{C} 16$ & $108.67(39)$ \\
\hline $\mathrm{C} 4-\mathrm{C} 3-\mathrm{C} 2$ & $110.17(59)$ & $\mathrm{C} 14-\mathrm{C} 15-\mathrm{C} 17$ & $113.76(50)$ \\
\hline $\mathrm{C} 7-\mathrm{C} 5-\mathrm{C} 4$ & $112.29(53)$ & $\mathrm{C} 16-\mathrm{C} 15-\mathrm{C} 17$ & $110.53(49)$ \\
\hline $\mathrm{C} 7-\mathrm{C} 5-\mathrm{C} 6$ & $111.12(68)$ & $\mathrm{C} 19-\mathrm{C} 18-\mathrm{C} 20$ & $111.44(53)$ \\
\hline $\mathrm{C} 4-\mathrm{C} 5-\mathrm{C} 6$ & $109.55(50)$ & $\mathrm{C} 19-\mathrm{C} 18-\mathrm{C} 12$ & $114.08(55)$ \\
\hline $\mathrm{C} 9-\mathrm{C} 8-\mathrm{C} 10$ & $105.47(82)$ & $\mathrm{C} 20-\mathrm{C} 18-\mathrm{C} 12$ & $111.09(47)$ \\
\hline $\mathrm{C} 9-\mathrm{C} 8-\mathrm{C} 2$ & $114.37(75)$ & $\mathrm{C} 12-\mathrm{C} 11-\mathrm{C} 16$ & $111.54(35)$ \\
\hline $\mathrm{C} 10-\mathrm{C} 8-\mathrm{C} 2$ & $111.85(58)$ & $\mathrm{C} 12-\mathrm{C} 11-\mathrm{Sn} 2$ & $113.71(22)$ \\
\hline $\mathrm{C} 5-\mathrm{C} 6-\mathrm{C} 1$ & $112.05(52)$ & $\mathrm{C} 16-\mathrm{C} 11-\mathrm{Sn} 2$ & $106.88(26)$ \\
\hline $\mathrm{F} 1-\mathrm{Sn} 1-\mathrm{C} 1$ & $96.59(12)$ & $\mathrm{C} 11-\mathrm{C} 12-\mathrm{C} 13$ & $110.04(31)$ \\
\hline $\mathrm{F} 1-\mathrm{Sn} 1-\mathrm{C} 1^{\mathrm{i}}$ & $96.58(2)$ & $\mathrm{C} 11-\mathrm{C} 12-\mathrm{C} 18$ & $113.47(36)$ \\
\hline $\mathrm{C} 1-\mathrm{Sn} 1-\mathrm{C} 1^{\mathrm{i}}$ & $118.72(12)$ & $\mathrm{C} 13-\mathrm{C} 12-\mathrm{C} 18$ & $113.33(43)$ \\
\hline $\mathrm{F} 1-\mathrm{Sn} 1-\mathrm{C} 1^{\mathrm{ii}}$ & $96.58(12)$ & $\mathrm{C} 14-\mathrm{C} 13-\mathrm{C} 12$ & $113.76(48)$ \\
\hline $\mathrm{C} 1-\mathrm{Sn} 1-\mathrm{C} 1^{\mathrm{ii}}$ & $118.72(16)$ & $\mathrm{C} 13-\mathrm{C} 14-\mathrm{C} 15$ & $110.99(49)$ \\
\hline $\mathrm{C}{ }^{\mathrm{i}}-\mathrm{Sn} 1-\mathrm{C}^{1 \mathrm{ii}}$ & $118.67(10)$ & $\mathrm{C} 11^{\mathrm{i}}-\mathrm{Sn} 2-\mathrm{C} 11^{\mathrm{ii}}$ & $116.91(5)$ \\
\hline $\mathrm{F} 2-\mathrm{Sn} 2-\mathrm{C} 11^{\mathrm{ii}}$ & $100.25(11)$ & $\mathrm{C} 11-\mathrm{Sn} 2-\mathrm{C} 11^{\mathrm{ii}}$ & $116.93(13)$ \\
\hline
\end{tabular}

Symmetry codes:

(i) 1-y, x-y, z; (ii) 1-x+y, 1-x, z. 